\title{
Imaging-guided revival of nanomedicine?
}

\author{
“...a pressing need exists to quantitatively \\ evaluate nanodrug performance at the cellular, \\ tissue and system level before advancement to \\ patients."
}

First draft submitted: 14 October 2016; Accepted for publication: 26 October 2016; Accepted for publication: 23 November 2016

Keywords: cancer • drug delivery • molecular imaging • nanoreporter • nanotherapy - personalized medicine $\bullet$ PET

Nanomedicine's ability to improve the pharmacokinetics, stability and toxicity profile of certain drugs, potentially augmenting their therapeutic index, has made it an attractive field of research. The first US FDA approved nanodrug became available for clinical cancer care in 1995. Nanomedicine is also being explored in a range of other diseases but despite its potential, successful stories of clinical translation are sparse [1,2]. Furthermore, disappointing results in recent clinical trials and the ensuing financial repercussions for the leading nanomedicine company [3] have compelled some to question nanomedicine's true value, potentially compromising the field's ability to translate promising future treatments. Certainly, the clinical translation of nanotherapies faces many challenges [4], with the inherent tumor heterogeneity found in human cancer $[5,6]$ standing out as a major obstacle toward a more widespread use. This variability causes nanotherapies' efficacy to vary vastly among patients - and among individual lesions within the same subject emphasizing the need for the development of patient amenability screening procedures.

The current debate about nanomedicine's delivery problems [7-10] can only be welcomed, and should help advance the field. However, not all nanodrugs are created equal and poor delivery may be the result of poor design, stability, pharmacokinetics or bioavailability, or simply due to intrinsic biological variability. Either way, a pressing need exists to quantitatively evaluate nanodrug performance at the cellular, tissue and system level before advancement to patients. To aid in this process, we believe that different molecular imaging strategies offer an attractive solution.

First, nanodrugs' complexity requires indepth investigations about in vivo behavior, with a focus on stability-determining drug-carrier and drug-blood interactions, especially for controlled release platforms [11]. In this scenario, noninvasive imaging, for example, allows evaluating drug-nanocarrier dynamics in vivo. The information derived from these experiments can be used to optimize nanoparticle formulation by increasing compatibility between drug and carrier to ultimately improve therapeutic results [11]. Moreover, imaging-guided screens can be implemented to evaluate nanoparticle performance at the cellular, tissue, organ and organism level and identify the most suitable carrier formulation for a given drug [12].

Second, radiolabeling of nanoparticles enables establishing their pharmacokinetics and tissue distribution ex vivo. Importantly, the use of certain radioisotopes also allows nanomaterials' in vivo visualization and longitudinal evaluation of their biodistribution using nuclear imaging techniques such as positron emission tomography (PET) and single photon emission computed tomography in a quantitative fashion at unlimited tissue penetration and uncontested sensitivity. Capital-

\section{Max L Senders}

Translational \& Molecular Imaging Institute, Icahn School of Medicine at Mount Sinai, New York, NY 10029, USA and

Department of Medical Biochemistry Academic Medical Center,

1105 AZ Amsterdam, The Netherlands

Zahi A Fayad

Translational \& Molecular Imaging Institute, Icahn School of Medicine at Mount Sinai, New York, NY 10029, USA

\section{Thomas Reiner}

Department of Radiology, Memorial Sloan-Kettering Cancer Center,

New York, NY 10065, USA

and

Department of Radiology, Weill Cornell Medical College, New York, NY 10065, USA

\section{Willem JM Mulder}

Translational \& Molecular Imaging Institute, Icahn School of Medicine at Mount Sinai, New York, NY 10029, USA and

Department of Medical Biochemistry, Academic Medical Center,

1105 AZ Amsterdam, The Netherlands

\section{Carlos Pérez-Medina}

Author for correspondence:

Translational \& Molecular Imaging Institute, Icahn School of Medicine at Mount Sinai, New York, NY 10029, USA Tel.: +1 2128249032

carlos.perez-medina@mountsinai.org 
izing on these features, we have recently introduced a new approach that revolves around the use of a separate nanoparticulate PET imaging entity - a nanoreporter - with similar characteristics to the nanotherapeutic [13]. We show that the PET nanoreporter approach does not require chemical modification of the clinical grade nanodrug, DOXIL, but can be co-administered at very low quantities, thus not affecting the therapeutic dose. In the recently published study, we observed the earlier mentioned heterogeneous tumor delivery rates, varying by more than one order of magnitude among individual animals. Using this PET nanoreporter technology in a mouse model of breast cancer, we were able to accurately determine DOXIL tumor accumulation noninvasively. Animals that had higher DOXIL concentrations, as determined by a single PET measurement, displayed significantly reduced tumor growth rates and prolonged survival as compared to those with lower concentrations. PET nanoreporter imaging therefore allows quantifying intratumoral nanodrug concentration in individual subjects, which is predictive of nanotherapy outcome.

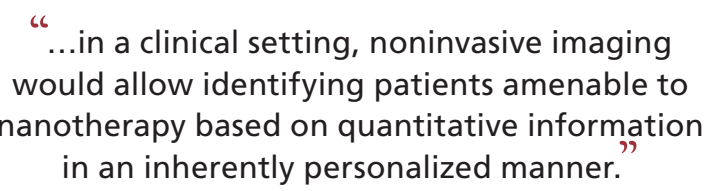

As nanoparticle tumor accumulation is mainly dictated by universally applicable mechanisms, one single clinically approved nanoreporter could potentially be used to monitor a range of different nanoparticle formulations. The therapeutic and economic implications of such screening protocols' implementation could be profound, as only one single imaging session would suffice to identify potential responders, saving precious

\section{References}

1 Juliano R. Nanomedicine: is the wave cresting? Nat. Rev. Drug Discov. 12, 171-172 (2013).

2 Venditto VJ, Szoka FC Jr. Cancer nanomedicines: so many papers so few drugs! Adv. Drug Deliv. Rev. 65, 80-88 (2013).

3 Ledford H. Bankruptcy filing worries developers of nanoparticle cancer drugs. Nature 533, 304-305 (2016).

4 Sanhai WR, Sakamoto JH, Canady R, Ferrari M. Seven challenges for nanomedicine. Nat. Nanotechnol. 3, 242-244 (2008).

5 Koren S, Bentires-Alj M. Breast tumor heterogeneity: source of fitness, hurdle for therapy. Mol. Cell 60, 537-546 (2015).

6 Asselin MC, O'Connor JP, Boellaard R, Thacker NA, Jackson A. Quantifying heterogeneity in human tumours using MRI and PET. Eur. J. Cancer 48, 447-55 (2012).

7 Torrice M. Does nanomedicine have a delivery problem? Chem. Eng. News 94, 16-19 (2016). time to nonresponders and enabling the pursuit of a different treatment option. Moreover, educated dose adjustments could be made with the help of this tool.

It is worth noting that while adequate drug delivery is a sine qua non, response to therapy is a more complex problem, as other factors influence final outcome. Even in cases when tumor accumulation would seem sufficient, a therapeutic effect may not be observed due to off-target cellular delivery within the tumor or to drug resistance. Thus, identification of patients amenable to nanotherapy by a nanoreporter-like method is just the first step toward a personalized process that could also include screening tests to identify potential drug resistance and treatment monitoring protocols to assess response, ideally in a noninvasive fashion. In this setting, the combined results from these tests should furnish information that would help to tailor a personalized treatment with increasing likelihood of success.

In summary, we here advocate the implementation of preclinical imaging-guided evaluation to optimize nanotherapeutics, based on their in vivo behavior, while in a clinical setting, noninvasive imaging would allow identifying patients amenable to nanotherapy based on quantitative information in an inherently personalized manner.

\section{Financial \& competing interests disclosure}

The authors have no relevant affiliations or financial involvement with any organization or entity with a financial interest in or financial conflict with the subject matter or materials discussed in the manuscript. This includes employment, consultancies, honoraria, stock ownership or options, expert testimony, grants or patents received or pending, or royalties. No writing assistance was utilized in the production of this manuscript.

8 Wilhelm S, Tavares AJ, Dai Q et al. Analysis of nanoparticle delivery to tumours. Nat. Rev. Mater. 1, 16014 (2016).

9 Lammers T, Kiessling F, Ashford M, Hennink W, Crommelin D, Storm G. Cancer nanomedicine: Is targeting our target? Nat. Rev. Mater. 1, 16069 (2016).

10 McNeil SE. Evaluation of nanomedicines: stick to the basics. Nat. Rev. Mater. 1, 16073 (2016).

11 Zhao Y, Fay F, Hak S et al. Augmenting drug-carrier compatibility improves tumor nanotherapy efficacy. Nat. Commun. 7, 11221 (2016).

12 Tang J, Baxter S, Menon A et al. Immune cell screening of a nanoparticle library improves atherosclerosis therapy. Proc. Natl Acad. Sci. USA 113, E6731-E6740 (2016).

13 Pérez-Medina C, Abdel-Atti D, Tang J et al. Nanoreporter PET predicts anti-cancer nanotherapy's efficacy. Nat. Commun. 7, 11838 (2016). 